\title{
Cytochrome P450 1A1 enhances inflammatory responses and impedes phagocytosis of bacteria in macrophages during sepsis
}

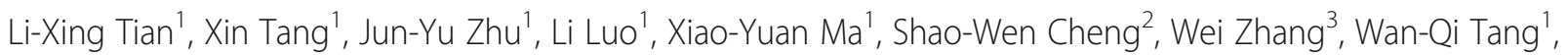 \\ Wei Ma', Xue Yang ${ }^{1}$, Chuan-Zhu Lv² and Hua-Ping Liang ${ }^{1 *}$
}

\begin{abstract}
The hydroxylase cytochrome P450 1A1 (CYP1A1) is regulated by the inflammation-limiting aryl hydrocarbon receptor (AhR), but CYP1A1 immune functions remain unclear. We observed CYP1A1 overexpression in peritoneal macrophages (PMs) isolated from mice following LPS or heat-killed Escherichia. coli (E. coli) challenge. CYP1A1 overexpression augmented TNF- $a$ and IL-6 production in RAW264.7 cells (RAW) by enhancing JNK/AP-1 signalling. CYP1A1 overexpression also promoted 12S-hydroxy-5Z,8Z,10E,14Z-eicosatetraenoic acid (12(S)-HETE) production in activated RAW, while a 12(S)-HETE antibody attenuated and 12(S)-HETE alone induced inflammatory responses. Macrophages harbouring hydroxylase-deficient CYP1A1 demonstrated reduced 12(S)-HETE generation and LPSinduced TNF-a/IL-6 secretion. CYP1A1 overexpression also impaired phagocytosis of bacteria via decreasing the expression of scavenger receptor A (SR-A) in PMs. Mice injected with CYP1A1-overexpressing PMs were more susceptible to CLP- or E. coli-induced mortality and bacteria invading, while Rhapontigenin, a selective CYP1A1 inhibitor, improved survival and bacteria clearance of mice in sepsis. CYP1A1 and 12(S)-HETE were also elevated in monocytes and plasma of septic patients and positively correlated with SOFA scores. Macrophage CYP1A1 disruption could be a promising strategy for treating sepsis.
\end{abstract}

Keywords: Cytochrome P450 1A1, Inflammation, Phagocytosis, Macrophages, Sepsis

\section{Background}

Sepsis is one of main causes of death in the intensive care unit. It occurs when the immune system becomes dysfunctional in response to existing infection and triggers a cascade of inflammatory responses [1]. Although there are a number of pharmacological interventions for curing sepsis, mortality remains unacceptably high [2]. Thus, promising targets and effective pharmacological treatments for septic patients are urgently needed. Emerging evidence suggests that inflammatory factors such as TNF- $\alpha$ and IL- 6 released by hyperactivated macrophages enhance the damaging responses during sepsis [3]. The

\footnotetext{
* Correspondence: 13638356728@163.com

${ }^{1}$ State Key Laboratory of Trauma, Burns and Combined Injury, Department of Wound Infection and Drug, Daping Hospital, Army Medical University, Yuzhong District, Chongqing, China

Full list of author information is available at the end of the article
}

Gram-negative bacterial LPS generally plays a beneficial role in controlling bacterial infections, but it is also a major driver of aberrant inflammatory factors production in lethal sepsis via binding to Toll Like Receptor 4 [4-6]. In addition, ingestion of bacteria in macrophages has been well-documented as a critical tache in host defence against pathogen invading [7]. Thus, it is essential to elucidate the fundamental mechanisms underlying LPS- and bacteria-induced macrophage overactivation and phagocytosis during sepsis.

CYP1A1 is involved in the metabolism of a broad spectrum of xenobiotics [8-10]. However, several investigations have reported that CYP1A1 also play critical roles in oxidative stress injury, as well as in mycoplasma and Citrobacter rodentium infection [11-17]. It has been reported that CYP1A1 is a critical enzyme mediating the metabolism of arachidonic acid (AA) to 12(S)-HETE 
through its hydroxylase activity [18-20]. AP-1, a transcriptional factor composed of c-jun and c-fos families, is a well-documented regulator of inflammatory responses by LPS-induced macrophages [21] and can also be activated by $12(\mathrm{~S})$-HETE [22-24]. It has been reported that TCDD, an AhR-related inducer of CYP1A1, enhances the DNA binding activity of AP-1 in normal Hepa cells, but not cells expressing hydroxylase-deficient CYP1A1 [25], suggesting a potential relationship between CYP1A1 and AP-1. However, no studies so far have investigated the relationships among CYP1A1, 12(S)-HETE and JNK/AP-1 in macrophages during inflammation or sepsis.

In this study, we identified CYP1A1 as a critical regulator of inflammatory responses and phagocytosis in sepsis and described two novel CYP1A1-invovled signalling pathways, CYP1A1-12(S)HETE-JNK - AP-1 and CYP1A1-SR-A, that may be promising targets for treating sepsis or other inflammatory diseases.

\section{Methods and materials Materials}

LPS (Escherichia coli 0111: B4) and PMA was purchased from Sigma-Aldrich. 12(S)-HETE from Cayman, and 12(S)-HETE-blocking antibody from ENZO 12(S)-HETE ELISA kit, JNK inhibitor SP600125, AP-1 inhibitor PNRI-299, 12-LOX inhibitor ML355 were produced by MedChemExpress. CYP1A1 inhibitor Rhapontigenin were produced by Santacruz. SR-A monoclonal antibody was purchased from Serotec. Penicillin, streptomycin, puromycin, RPMI 1640 and foetal bovine serum (FBS) were obtained from Gibco-BRL Invitrogen. Ficoll Paque PLUS was purchased from GE Healthcare Life Sciences.

\section{Preparation of E.coli cells}

E.coli cells (25922, ATCC) were seeded on LB agar plates and cultured at $37^{\circ} \mathrm{C}$ for commonly maintaining in our lab. One colony from these growing LB agar plates were transplanted into $100 \mathrm{ml}$ of fresh sterile LB medium and incubated on a orbital shaker at $37{ }^{\circ} \mathrm{C}$ for $12 \mathrm{~h}$ and then transferred to $500 \mathrm{ml}$ of fresh sterile $\mathrm{LB}$ medium for another $12 \mathrm{~h}$. The viable E.coli cells were harvested by centrifugation at $10000 \times \mathrm{g}$ for $5 \mathrm{~min}$ and washed by $0.9 \% \mathrm{NaCl}$ sterile solution, and then resuspended by sterile glycerine. The E.coli cells were incubated in a water bath at $90{ }^{\circ} \mathrm{C}$ for $15 \mathrm{~min}$ for inactivation (heat kill).

\section{Mice}

Healthy C57BL/6 mice (male, 10-12 weeks, 20-25 g) were provided by the Experimental Animal Center of Army Medical University (Chongqing, China). AhR ${ }^{+/-}$ mice, inbred C57BL/6 back-ground, were born and raised in indoor barrier maintained animal facilities at The Jackson Laboratory. WT and $\mathrm{AhR}^{-/-}$were bred from
$\mathrm{AhR}^{+/-}$mice and raised in isolation with Specific Pathogen Free status. All experimental procedures and animal welfare protocols were conducted in accordance with the guidelines for laboratory animal care of the National Institutes of Health and Army Medical University.

\section{In vitro culture}

\section{Mice peritoneal macrophages}

Healthy C57BL/6 mice were intraperitoneal injected with $4 \%$ thioglycolate for cell extraction. After 3 days' stimulation, macrophages were extracted from mice by douching the peritoneal cavity with $5 \mathrm{ml}$ cold phosphate buffer saline (PBS). Total extracted cells were centrifuged for $5 \mathrm{~min}$ at $300 \mathrm{~g}$ and seeded onto Petri dishes for $3 \mathrm{~h}$ at $37{ }^{\circ} \mathrm{C}$. Non-adherent cells were removed by washing with $\mathrm{PBS}$, and the adherent cells were harvested for future experiments.

\section{RAW264.7 cell line}

RAW264.7 cells (ATCC) were cultured in PRMI 1640 medium supplemented with $100 \mathrm{mg} / \mathrm{ml}$ streptomycin, $100 \mathrm{U} / \mathrm{ml}$ penicillin and $10 \% \mathrm{FBS}$ at $37{ }^{\circ} \mathrm{C}$ under a $5 \%$ $\mathrm{CO}_{2}$ / sterile air atmosphere. RAW264.7 cells were stably transfected with six types of recombinant lentivirus (GeneChem): 1. Lentivirus containing the whole coding sequences of CYP1A1 and enhanced (E)GFP. 2. Lentivirus containing the sequences of a hydroxylase-deficient CYP1A1 mutant and EGFP. 3. Lentivirus encoding a JNK CRISPR/CAS9 knockout system. 4. Lentivirus encoding a c-fos/c-jun CRISPR/CAS9 knockout system. 5. Lentivirus encoding a JNK CRISPR/CAS9 knockout system and CYP1A1-EGFP. 6. Lentivirus encoding a c-fos/ c-jun CRISPR/CAS9 knockout system and CYP1A1 -EGFP. The CYP1A1 hydroxylase mutant form was based on the c37 mutant used in previous study [26]. Successfully transfected cell lines were selected and maintained in the presence of $4 \mu \mathrm{g} / \mathrm{ml}$ puromycin.

\section{Isolation of primary peripheral monocytes}

Fresh blood was collected from consenting healthy and septic donors according to an institutional review board protocol approved by the ethics committee of Army Medical University or E.coli-treated mice, respectively. Whole blood was diluted 1:2 in PBS, layered over the lymphosep density separation medium Ficoll Paque PLUS, and concentrated by centrifugation for $20 \mathrm{~min}$ at 200 g. Mononuclear cells were washed 3 times with HEPES to remove platelets, isolated using a negative depletion kit (Invitrogen), and then maintain at $37{ }^{\circ} \mathrm{C}$ under a $5 \% \mathrm{CO}_{2} /$ sterile air atmosphere. Some isolated human peripheral monocytes were coaxed into macrophages by PMA $(100 \mathrm{ng} / \mathrm{ml})$ for $24 \mathrm{~h}$ for indicated experiments. 


\section{Quantitative reverse transcription $P C R$}

The total RNA was isolated from treated cells using TRIzol reagent (Invitrogen). $1 \mathrm{~g}$ cDNA was synthesized from quantified RNA according to the manufacturer's recommendations (TaKaRa) and the target mRNAs were quantified by qRT-PCR using SYBR Premix (TaKaRa) on a BioRad CFX96. All primer sequences used in this study are listed in supplemental table 1.

\section{ELISA}

The concentrations of TNF- $\alpha$ and IL- 6 in the cell supernatants, PLF and plasma were determined by ELISA kits (Boster) according to the manufacturer's instructions (450 nm absorbance). The levels of 12(S)-HETE in above samples were measured using a 12(S)-HETE ELISA kit (Cayman) at $405 \mathrm{~nm}$ absorbance. The level of 14, 15EET was assessed using ELISA kit (Detroit R\&D) following the manufacture's instruction.

\section{Aspartate Transaminase (AST) and Alanine Transaminase (ALT) Measurements}

The blood samples were separated by centrifugation (300 g, $10 \mathrm{~min}$ ) and the serum portion was sent to the Department of Laboratory Medicine, Daping Hospital, Army Medical University, for quantification of AST and ALT.

\section{Western blotting}

Treated cells were lysed on ice for $30 \mathrm{~min}$ and the supernatant cleared by centrifugation at $12000 \mathrm{~g}$ for $15 \mathrm{~min}$ at $4{ }^{\circ} \mathrm{C}$. Equal amounts of protein per gel lane were separated by $10 \%$ SDS-PAGE and transferred to PVDF membranes. Membranes were blocked with 5\% skim milk for $1 \mathrm{~h}$ in Tris-buffered saline containing $0.1 \%$ Tween 20 (TBST) at room temperature (RT), and then incubated overnight at $4{ }^{\circ} \mathrm{C}$ in primary antibodies. The blotted membranes were washed 3 times with TBST and probed for $1 \mathrm{~h}$ with appropriate HRP-conjugated secondary antibodies (Cell Signalling Technology). The immunoreactive bands were visualized using an enhanced chemiluminescence detection system (Bio-Rad). The relative intensities of bands were measured by ImageJ software. CYP1A1, 12-LOX, phosphorylated-p50, p50, phosphorylated-JNK and total JNK antibodies were purchased from Abcam. Antibodies against phosphorylated-c-jun, phosphorylated-c-fos, phosphorylated-p65, phosphorylatedERK $_{1 / 2}$, phosphorylated-p38, c-jun, c-fos, p65, ERK $K_{1 / 2}$, p38, GPADH and $\beta$-actin were purchased from Cell Signalling Technology. All antibodies mentioned above are diluted 1:1000 for western blot analysis.

\section{Target protein knockdown by RNA interference}

CYP1A1 and scrambled control siRNA were provided by GenePharma. Macrophages were seeded at $2 \times 10^{6}$ cells per well (CYP1A1/RAW cells were seeded at $5 \times 10^{5}$ cells per well) on six-well plates and incubated with $120 \mathrm{nM}$ scramble siRNA or target siRNA in Lipo2000 Reagent (GenePharma) for $8 \mathrm{~h}$. The transfection medium was removed and replaced by fresh RPMI 1640 containing $12 \%$ serum. After $48 \mathrm{~h}$ transfection, cells were then stimulated with 12(S)-HETE or LPS as indicated times.

\section{Electrophoretic mobility shift assay}

Nuclear proteins were extracted from treated cells using nuclear and cytoplasmic protein extraction kits (Beyotime) following standard procedures. Binding of nuclear extracts to AP-1-binding sites was assessed by electrophoresis of equal amounts of protein and DNA-protein complex on $6 \%$ polyacrylamide native gels for $50 \mathrm{~min}$ at $160 \mathrm{~V}$. Then the gels were detected at $700 \mathrm{~nm}$ using an Odyssey scanning bed (LiCor). The consensus binding sequence for AP-1 was 5'-CGCTTGATGACTCAGCCGGAA-3'.

\section{Laser scanning confocal microscopy}

Primary monocytes were isolated according to the protocol described above. Fresh cells were plated on coverslips (Nest) and fixed in $4 \%$ paraformaldehyde (Beyotime)/PBS at RT for $30 \mathrm{~min}$, permeabilized with $0.5 \%$ Triton X-100 (Beyotime) /PBS at RT for $10 \mathrm{~min}$, followed by blocking with PBS containing $1 \%$ bovine serum albumin (BSA) and 1\% rabbit serum for $30 \mathrm{~min}$. Fixed cells were incubated with primary antibody against CYP1A1 (1:250, CST), phosphorylated JNK (p-JNK, 1: 100, CST), phosphorylated c-jun (1:100, CST) and phosphorylated c-fos $(1: 100, \mathrm{CST})$ at $4{ }^{\circ} \mathrm{C}$ overnight, followed by Alexa Fluor 594-conjugated donkey secondary antibody (1:200, Invitrogen) in the dark for $2 \mathrm{~h}$ in PBS containing 1\% BSA and $0.5 \%$ Triton X-100. Cells were then counterstained with DAPI (Beyotime) for $10 \mathrm{~min}$. After incubation, all coverslips were washed three times in PBS and imaged using a LSM 780 laser-scanning confocal microscope (Carl Zeiss).

\section{Inhibitor assays}

In vitro

RAW264.7 cells were seeded at $2 \times 10^{5}$ cells/well in sixwell plates and cultured for $24 \mathrm{~h}$ in RPMI 1640. PMs were seeded at $1 \times 10^{6}$ cells/well. Cells were then pretreated with vehicle, 12(S)-HETE antibody $(3 \mu \mathrm{g} / \mathrm{ml}, 12$ $\mathrm{h}$ before challenge), ML355 (10 $\mu \mathrm{M}, 2 \mathrm{~h}$ before challenge) or SR-A monoclonal antibody $(3 \mu \mathrm{g} / \mathrm{ml}, 12 \mathrm{~h}$ before challenge) as indicated then treated with vehicle, LPS $(10 \mu \mathrm{g} / \mathrm{ml})$ or E.coli (MOIs=30) for the indicated times. 


\section{In vivo}

C57BL/6 mice (male, 10-12 weeks, 20-25 g) were fasted for $12 \mathrm{~h}$ and then pre-treated with vehicle, JNK inhibitor (30 mg/kg) or AP-1 inhibitor $(20 \mathrm{mg} / \mathrm{kg}$ ) for $2 \mathrm{~h}$. Mice were then treated with vehicle or $E$. coli $\left(1.2 \times 10^{11}\right.$ $\mathrm{CFUs} / \mathrm{kg}$ ) for the indicated times. In survival experiments, JNK and AP-1 inhibitors were re-injected 6, 12 and $24 \mathrm{~h}$ after E. coli or CLP challenge. Plasma was isolated from mouse venous blood for various markers assays as described.

\section{CYP1A1 hydroxylase activity assay}

Benzo $[\alpha]$ pyrene is frequently used as a substrate for assessing CYP1A1 hydroxylase activity. The measurement was consulted with previous studies [26, 27].

\section{Macrophages infection with adenovirus}

PMs were seeded at $2 \times 10^{6}$ cells per well on six-well plates and infected with 1000 MOIs negative control adenovirus, adenovirus encoding CYP1A1-GFP or adenovirus encoding a hydroxylase-deficient CYP1A1 -GFP for $8 \mathrm{~h}$, then cultured for $40 \mathrm{~h}$ in fresh RPMI 1640. After $48 \mathrm{~h}$ infection, the macrophages were harvested for Western blot analysis.

\section{Sepsis models}

CLP was applied to induce the polymicrobial sepsis model as previous study described [28]. Mice were injected intraperitoneally with $1.2 \times 10^{11} \mathrm{CFU} / \mathrm{kg}$ E. coli to induce the monomicrobial sepsis [29]. Vector-infected PMs $\left(5 \times 10^{6}\right.$ cells per mouse $)$ were injected intraperitoneally into WT mice 2 days and Rhapontigenin were injected intraperitoneally into WT mice $1 \mathrm{~h}$ before $E$. coli- or CLP-induced sepsis and survival monitored for the indicated periods. Some treated mice were sacrificed at $12 \mathrm{~h}$ after E.coli or CLP challenge for sample collection. PLF was prepared by a single washing of the peritoneal cavity with $3 \mathrm{ml}$ cold PBS. PLF, Spleen and liver were harvested for bacterial burden detection. The 12(S)-HETE, TNF- $\alpha$ and IL-6 concentrations were determined by ELISA and bacterial burden was measured by bacterial colony count in PLF. Serum from indicated septic mice was also extracted for ALT and AST assays. In phagocytosis experiments, vector-infected PMs were injected intraperitoneally into WT mice 30 minutes before $E$. coli onset or 0 minute before CLP onset. Some treated mice were sacrificed at 40 minutes after E.coli or CLP challenge for macrophages SR-A mRNA and intracellular bacteria count measurements.

\section{Detection of bacterial burden}

In vivo experiments, isopyknic PLFs (3 $\mathrm{ml}$ for each), spleen and liver were collected from indicated septic mice after 12 h E.coli or CLP challenge. PLFs were diluted 1000-fold with $1 \mathrm{ml}$ sterile LB. Equal amounts of tissues were homogenized in $3 \mathrm{ml}$ sterile LB in ice and lapping tissues fluids were diluted 500-fold with $1.5 \mathrm{ml}$ sterile LB. CYP1A1-modified, Rhapontigenin-treated PMs, extracted PMs from Ad-CYP1A1 macrophages transferred or Rhapontigenin treated septic mice were harvested after 40 minutes impact. The harvested cells were centrifuged for $5 \mathrm{~min}$ at $300 \mathrm{~g}$ and suspended with $1 \mathrm{ml}$ sterile LB. The suspensions were diluted 100-fold with $1 \mathrm{ml}$ sterile LB. Each dilution was plated on LB agar plate at $200 \mu \mathrm{l}$ volume respectively and incubated at 37 ${ }^{\circ} \mathrm{C}$ under sterile air atmosphere for $18 \mathrm{~h}$. The numbers of bacterial colonies were calculated as CFUs.

\section{Study subjects}

From November 2017 to December 2017 and November 2018 to December 2018, thirty eligible patients were screened within $48 \mathrm{~h}$ after admission to the ICU of the Third Affiliated Hospital of Army Medical University. Patients were enrolled if they had known or suspected infection plus a SOFA score of 2 or more for organ dysfunction and the ability to provide informed consent. Exclusion criteria were age younger than 17 or older than 76 years, mental disorders, or sepsis combined with other severe somatic diseases (malignant tumour or cancer). The clinical data are summarized in supplemental table 2. Thirty healthy individuals in the medical examination centre of the Third Affiliated Hospital of Army Medical University were also enrolled as controls. The study protocol was approved by the local institutional review board, and informed consent was obtained from all subjects or their surrogates. Fresh plasma was isolated from peripheral venous blood (patients were fasted) on day 2 of ICU admission. Monocytes were prepared as previously described. Expression levels of CYP1A1 mRNA were assessed using qRT-PCR. The plasma (diluted 1:100 in assay buffer) concentration of 12(S)-HETE was determined by ELISA. Expression levels of CYP1A1, phosphorylation levels of JNK and AP-1 in monocytes were observed using laser scanning confocal analysis.

\section{Statistical analysis}

Most data are presented as the mean \pm standard error (SEM) of three independent experiments. Multiple group means were compared by one-way ANOVA followed by LSD multiple-comparison tests. Survival rates were analysed using the Mantel-Cox test. Categorical data were compared between two groups by chi-squared test. Continuous variables were compared between two groups by two-tailed Student's $t$-test. Correlations with SOFA scores were analysed by linear regression. All statistical analyses were performed using SPSS 16.0 (SPSS Inc.) or Prism 6.0 (GraphPad software Inc.) software, and a Pvalue $<0.05$ was considered statistically significant. 


\section{Results}

\section{CYP1A1 is upregulated in PMs of septic mice}

In current study, we found that CYP1A1 was significantly elevated in PMs isolated from LPS- or E. coli-induced mice (Fig. 1a). The levels of CYP1A1 mRNA in PMs were elevated at $2 \mathrm{~h}$ and reached a peak at $6 \mathrm{~h}$ after heat-killed E. coli or LPS treatment, while CYP1A1 protein expression was significantly upregulated at $12 \mathrm{~h}$ after challenge (Fig. 1b). AhR is the main regulator of CYP1A1 expression [30]. We found that CYP1A1 protein levels were still highly overexpressed in PMs from E.coli-treated AhR knockout $\left(\mathrm{AhR}^{-/-}\right)$mice (Fig. 1c), accompanied by TNF- $\alpha$ and IL- 6 in peritoneal lavage fluids (PLFs) increase (Fig. 1d). However, there were no differences in PMs counts between E.coli-treated $\mathrm{AhR}^{-/-}$mice and E.coli-treated wild-type (WT) mice (Fig. 1e). These results suggest a possible $\mathrm{AhR}$-independent regulation of CYP1A1 in the progression of inflammation and sepsis.

CYP1A1 is involved in LPS-induced macrophages activation To directly assess the function of CYP1A1 in regulating inflammatory factor expression, we established a cell line derived from RAW constitutively expressing CYP1A1 (CYP1A1/RAW) and a negative control line (NC/RAW) (Supplemental Figure 1 A). These CYP1A1/RAW exhibited significantly greater expression of TNF- $\alpha$ and IL- 6 at both mRNA and protein levels following LPS challenge than LPS-treated NC/RAW (Fig. 2a), while expression levels of IL-1 $\beta$ and NOS2 mRNA were not altered. We found that mRNA levels of Arg-1 and PPAR $\gamma$, two markers of M2 macrophage, were reduced in LPS- stimulated CYP1A1/RAW compared with LPS-stimulated NC/RAW (Supplemental Figure 2), suggesting that CYP1A1 may inhibit M2 macrophage polarization during LPS-induced inflammation. To confirm these regulatory effects of CYP1A1 on TNF- $\alpha$ and IL- 6 expression, we also examined LPS responses in macrophage transfected with a CYP1A1-targeting short interfering RNA (CYP1A1 siRNA) or scramble siRNA (Scr control). As CYP1A1 expression levels are too low to implement gene silencing in RAW, we instead conducted these experiments in mice PMs (Supplemental Figure 1 B). Consistent with overexpression experiments, expression levels of IL- 6 and TNF- $\alpha$ were reduced in CYP1A1 knockdown PMs following LPS treatment (Fig. 2b). Moreover, pre-treatment of PMs with a selective CYP1A1 enzymatic inhibitor Rhapontigenin [31, 32] following by LPS stimulation reduced IL- 6 and TNF- $\alpha$ levels compared to vehicle pre-treatment (Supplemental Figure 3). Interestingly, we found that LPS-stimulated TNF$\alpha$ and IL- 6 expression levels were still lower in CYP1A1 silent $\mathrm{AhR}^{-/-} \mathrm{PMs}$ compared to Scr control transfected $\mathrm{AhR}^{-/}$PMs, suggesting that CYP1A1-induced proinflammatory responses are also AhR-independent (Fig. 2c).

MAPK/AP-1 and NF- $\mathrm{B}$ are two main pathways of TNF- $\alpha$ and IL-6. Overexpression of CYP1A1 significantly enhanced phosphorylation of AP-1 and its upstream regulator JNK following LPS, while CYP1A1 knockdown reduced these phosphorylation responses (Fig. 2d). Alternatively, there were no differences in phosphorylation levels of p-38 between LPS-stimulated CYP1A1/RAW and NC/RAW, while the phosphorylation levels of NF- $\mathrm{B}$ and $E R K_{1 / 2}$ exhibited a moderate decrease
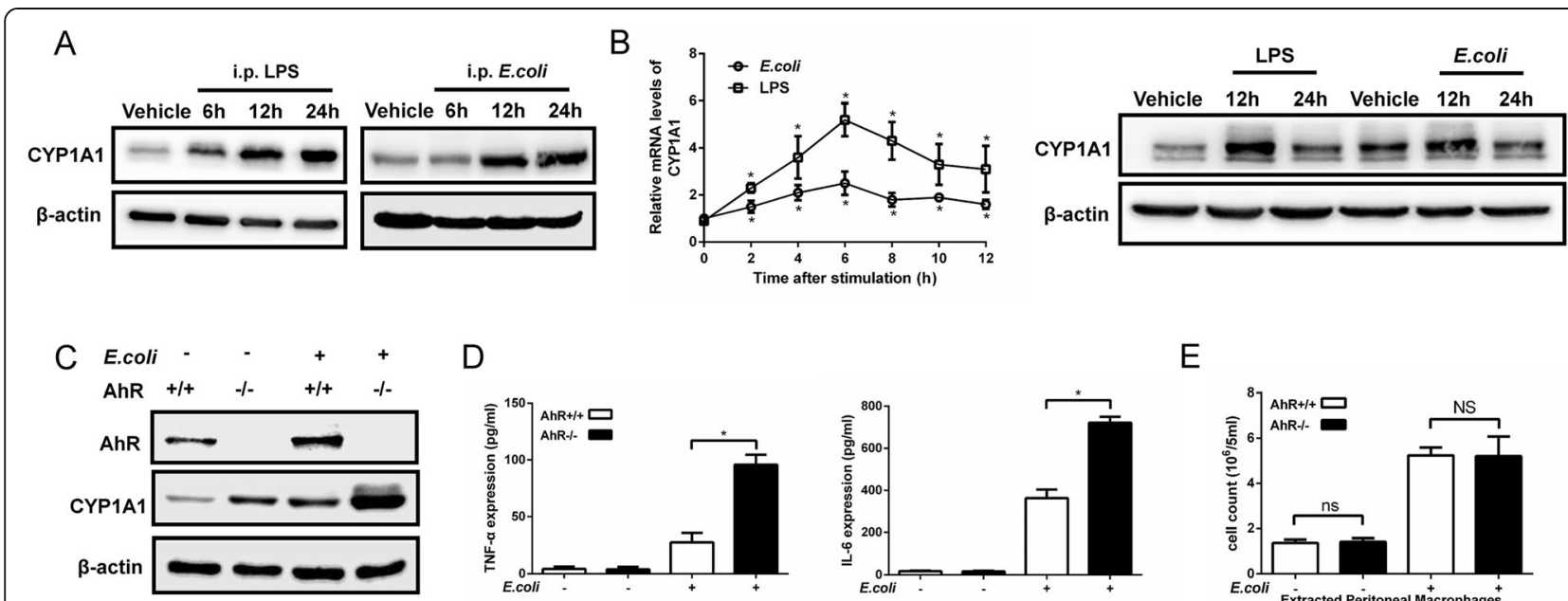

$\mathrm{D}$

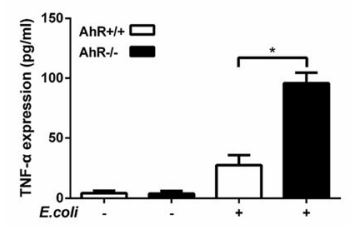

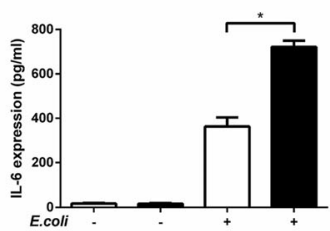

E

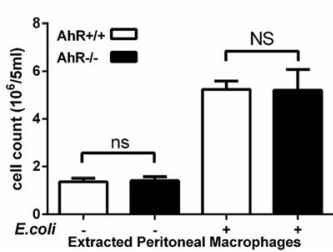

Fig. 1 CYP1A1 is upregulated in PMs of septic mice. a Mice were intraperitoneally injected with vehicle (isopyknic PBS), LPS (20 mg/kg) or E. coli $\left(1.2 \times 10^{11} \mathrm{CFUs} / \mathrm{kg}\right.$, CFUs, colony forming units). PMs were extracted at the indicated times and subjected to western blotting analysis of CYP1A1 protein levels. b PMs isolated from WT mice were treated with vehicle, LPS $(10 \mu \mathrm{g} / \mathrm{ml})$ or heat-killed E. coli (MOls $=10$, MOls, multiplicity of infections) for the indicated times. CYP1A1 mRNA expression was quantified by qRT-PCR. Expression levels of CYP1A1 protein were detected by western blotting. c-e AhR ${ }^{-/-}$and WT mice were intraperitoneally injected with vehicle or E.coli. After $12 \mathrm{~h}$ treatment, PMs and PLFs were extracted and subjected to analysis of AhR and CYP1A1 protein expression levels (a), pro-inflammatory cytokines expression levels (b) and PMs count (c). Data are mean \pm SEM of three independent experiments. Results were compared by one-way ANOVA. ${ }^{*} p<0.05$. NS, no statistical difference 


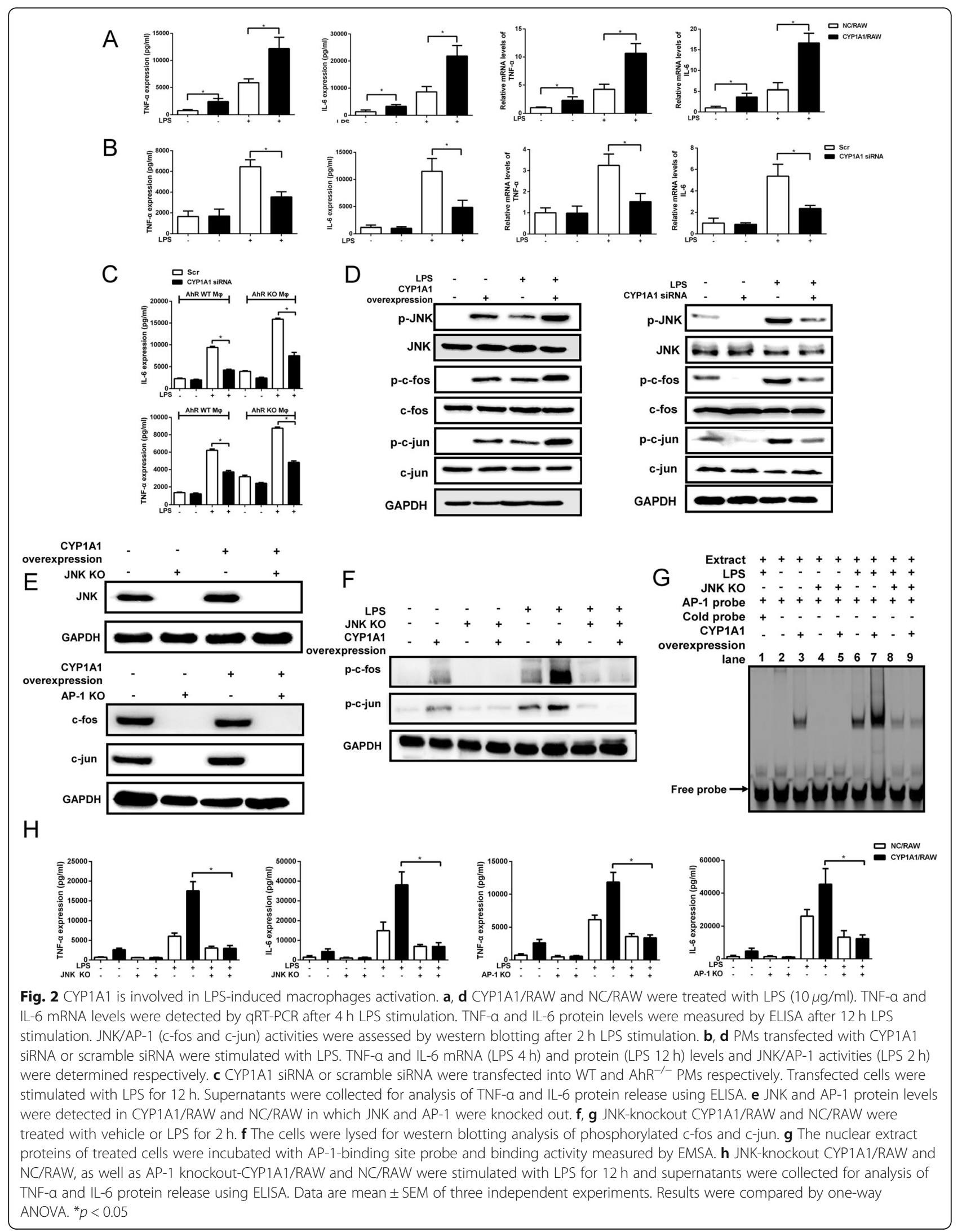


in LPS-induced CYP1A1/RAW compared to LPS-induced NC/RAW (Supplemental Figure 4). To directly demonstrate the role of JNK/AP-1 in CYP1A1-associated TNF- $\alpha$ and IL-6 expression, we knocked out JNK and AP-1 in NC/RAW and CYP1A1/RAW, respectively (Fig. 2e). LPSinduced AP-1 phosphorylation was decreased in JNK knockout CYP1A1/RAW compared whit control CYP1A1/RAW (Fig. 2f). The DNA-binding activity of AP1 was also greatly reduced by JNK knockout (Fig. 2g). Likewise, LPS-stimulated TNF- $\alpha$ and IL-6 expression levels were lower in both JNK and AP-1 knockout CYP1A1/RAW compared with control CYP1A1/RAW (Fig. 2h). Collectively, these data strongly suggest that CYP1A1 upregulates LPS-induced TNF- $\alpha$ and IL-6 expression by strengthening JNK-mediated AP-1 activation.

\section{CYP1A1-derived 12(S)-HETE production intensifies JNK/ AP-1 activation}

12(S)-HETE can activate AP-1 [22-24], so we further examined if CYP1A1 promotes LPS-induced inflammatory responses through 12(S)-HETE. Our data showed there is a positive correlation between CYP1A1 and 12(S)HETE production, which is associated with CYP1A1 hydroxylase activity (Fig. 3a). Moreover, direct treatment of RAW with 12(S)-HETE and LPS resulted in greater JNK/AP-1 phosphorylation and TNF- $\alpha$ and IL-6 expression compared to vehicle- and LPS-treated control RAW (Fig. $3 \mathrm{~b}$ and c). To further confirm that the inflammatory response caused by CYP1A1 overexpression is mediated by 12 (S)-HETE, we used a 12(S)-HETE-blocking antibody to neutralize 12(S)-HETE in LPS-stimulated CYP1A1/ RAW and NC/RAW. Indeed, this treatment abolished JNK/AP-1 activation and TNF- $\alpha$ and IL- 6 elevation associated with CYP1A1 overexpression (Fig. 3d-f). These results suggest that CYP1A1 controls the activation of macrophages in response to LPS via 12(S)-HETE generation.

\section{Elevation of 12(S)-HETE in LPS-stimulated CYP1A1/RAW cells is CYP1A1 hydroxylase-dependent rather than 12 lipoxygenase-dependent}

It was demonstrated that 12(S)-HETE also benefits from 12lipoxygenase (12-LOX) [33, 34]. However, overexpressedCYP1A1 failed to affect 12-LOX expression at either the mRNA or protein level (Fig. 4a). Furthermore, treatment of CYP1A1/RAW and NC/RAW with the specific 12-LOX

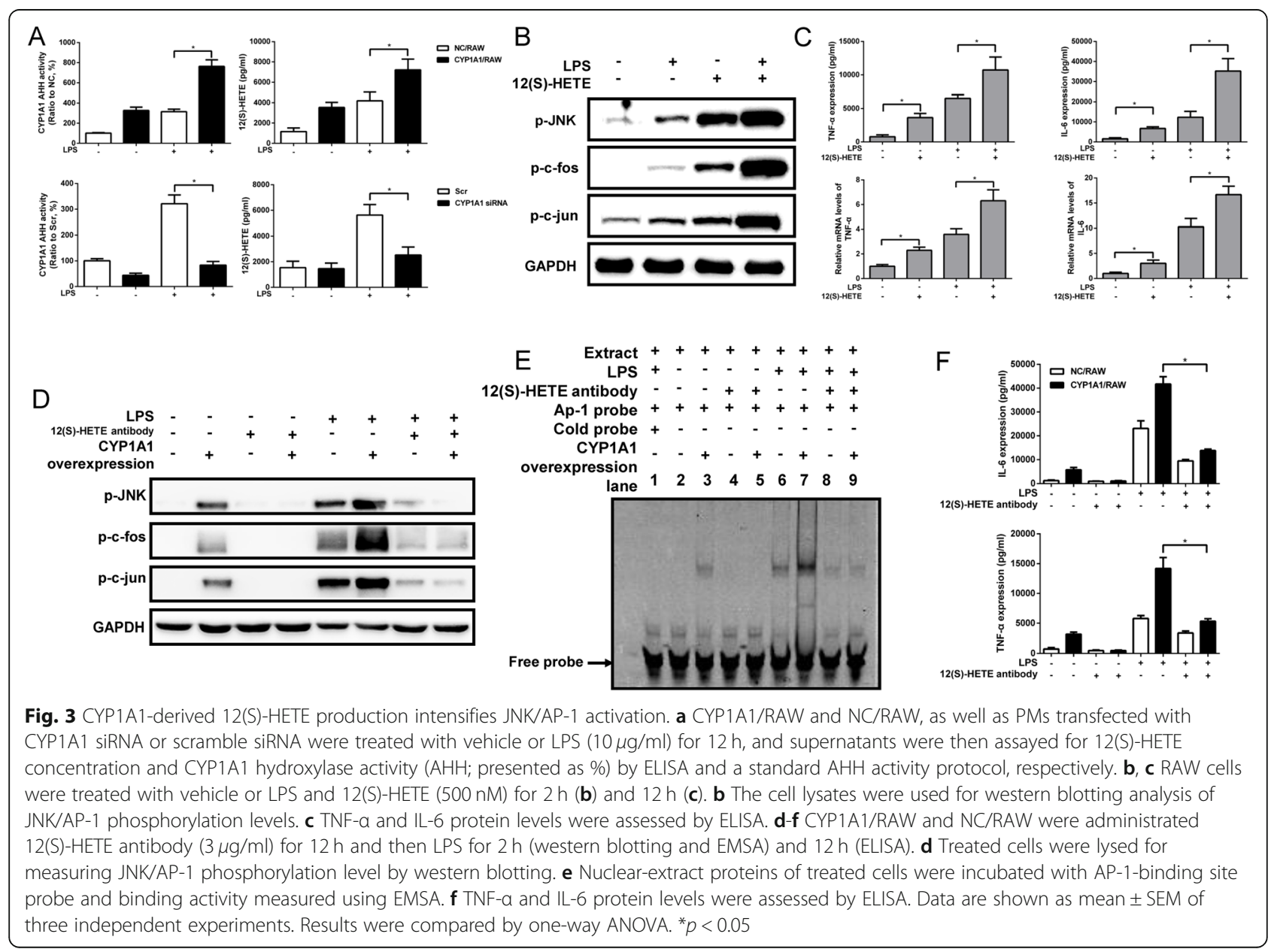


inhibitor ML355 [35] had no effect on CYP1A1 hydroxylase activity (Fig. 4b) and failed to abolish the production of 12(S)-HETE, TNF- $\alpha$, and IL- 6 by CYP1A1/RAW and NC/ RAW following LPS stimulation (Fig. 4c). It has been reported that CYP1A1 can metabolise AA to epoxyeicosatrienoic acids (EETs) by epoxidase activity as well as to HETEs by hydroxylase activity [19]. To confirm the proinflammatory effect caused by CYP1A1 was mainly due to its hydroxylase activity, we established a RAW cell line constitutively expressing a hydroxylase-deficient CYP1A1 (CYP1A1 mutant/RAW) according to previous research [32] (Fig. 4d). Disruption of CYP1A1 hydroxylase activity had no effect on CYP1A1 protein expression (Fig. 4e). 14, 15-EET, a representative EET produced by CYP1A1 [19], was not affected by CYP1A1 hydroxylase deficiency (Fig. 4h), indicating the mutant form we used is hydroxylasespecific. However, JNK/AP-1 phosphorylation and AP-1 DNA binding activity were reduced in CYP1A1 mutant/ RAW compared with normal CYP1A1/RAW after LPS stimulation (Fig. $4 \mathrm{f}$ and g). Furthermore, secretion of 12(S)HETE, TNF- $\alpha$ and IL- 6 were reduced (Fig. $4 \mathrm{~h}$ ). Collectively, these findings indicate that CYP1A1 upregulates the LPSinduced inflammatory response of macrophages through concomitant overproduction of 12(S)-HETE via CYP1A1 hydroxylase activity rather than 12 lipoxygenase activity.

\section{Regulation of the CYP1A1-12(S)HETE-JNK - AP-1 signalling axis in septic mice}

After CLP or E. coli impact, we found that the levels of 12(S)-HETE were elevated in mice PLFs (Supplemental Figure 5). JNK/AP-1 phosphorylation was also increased in PMs extracted from E. coli-challenged WT mice (Fig. 5a). Consistent with in vitro results, intraperitoneal injection of 12(S)-HETE elevated the concentrations of TNF- $\alpha$ and IL- 6 in mice PLFs, while JNK and AP-1 inhibitors abolished these effects (Fig. 5b). Furthermore, we isolated and cultured the peripheral monocytes from E. coli-treated mice to investigate the direct relationship among 12(S)-HETE, CYP1A1 and monocytes. The levels of 12(S)-HETE in monocytes cultured supernatants and levels of CYP1A1 in monocytes were elevated after $E$. coli treatment, respectively (Fig. 5c). The activation of JNK and c-jun in peripheral monocytes from E. colitreated mice was also confirmed by laser scanning confocal microscopy (LSCM) (Fig. 5d).

To specifically assess the involvement of CYP1A1 in sepsis, recombinant CYP1A1-expression adenovirus (AdCYP1A1), hydroxylase-deficient CYP1A1 adenovirus (AdCYP1A1m) and negative control adenovirus (Ad-NC) were constructed and transfected into WT PMs (Supplemental Figure $6 \mathrm{~A}$ ) and then evaluated the effects of these cells on the mortality of $E$. coli- or CLP-induced septic WT mice by adoptive cell transfer (Supplemental Figure 6 B). Compared to Ad-NC PMs injection, Ad-CYP1A1 PMs injection significantly accelerated the mortality and increased the expression levels of pro-inflammatory biomarkers, including ALT, AST, TNF- $\alpha$, IL- 6 and 12(S)-HETE, of mice in the $E$. coli- or CLP-induced septic mice (Fig. 5e and g), while AdCYP1A1m PMs injection had no these effects. Furthermore, treatment of mice with JNK and AP-1 inhibitors prior to Ad-NC and Ad-CYP1A1 PMs adoptive transfer decreased mice mortality and pro-inflammatory biomarkers in the E. coli- or CLP-induced sepsis model (Supplemental Figure 7). On the other hand, intraperitoneal injection of Rhapontigenin increased the survival and decreased pro-inflammatory biomarkers of mice in E. coli- or CLP-induced sepsis model (Fig. $5 \mathrm{f}$ and $\mathrm{h}$ ). As an important source of 12(S)-HETE, our data showed that platelet counts are abundantly increased in the peritoneal cavity following LPS, E.coli, or sepsis impact, while CYP1A1 overexpression in PMs was incapable to recruit platelets in PLFs (Supplemental Figure 8). Altogether, these findings confirmed the regulatory effects of CYP1A1/12(S)-HETE/ JNK/AP-1 axis in vivo, while Rhapontigenin reduces the mortality and pro-inflammatory biomarkers from sepsis.

\section{CYP1A1 is involved in phagocytosis of bacteria in macrophages during sepsis}

In current study, we observed a decrease in the count of intracellular bacteria in CYP1A1-overexpressing PMs following $40 \mathrm{~min}$ E.coli challenge, while the count of intracellular bacteria was increased in CYP1A1 knockdown and Rhapontigenin-treated PMs, respectively. However, direct treatment of PMs with 12(S)-HETE failed to change the count of intracellular bacteria in E.coli-treated PMs (Fig. 6a). SR-A is a critical controller of ingesting E.coli in macrophages [36]. Overexpression of CYP1A1 decreased the expression level of SR-A following E.coli treatment, while CYP1A1 knockdown and Rhapontigenin treatment elevated the expression levels of SR-A. Direct treatment of PMs with 12(S)-HETE had no effect on the expression of SR-A (Fig. 6b-d).

To directly demonstrate the role of SR-A in CYP1A1invovled bacteria ingestion, we used SR-A antibody in CYP1A1 silent and Rhapontigenin-treated PMs. The count of intracellular bacteria was significantly reduced in SR-A blocked PMs compared with control cells (Fig. 6e). Consistent with in vitro experiments, we observed decreased count of intracellular bacteria and mRNA levels of SR-A in PMs extracted from septic mice transferred with Ad-CYP1A1 PMs (Supplemental Figure 9 A, C), while the count of survival bacteria was elevated in PLFs (Supplemental Figure 9 B, D). In contrast, the count of intracellular bacteria and mRNA levels of SR-A were elevated in PMs extracted from septic mice treated with Rhapontigenin (Supplemental Figure 9 E, G), while the count of survival bacteria was decreased 


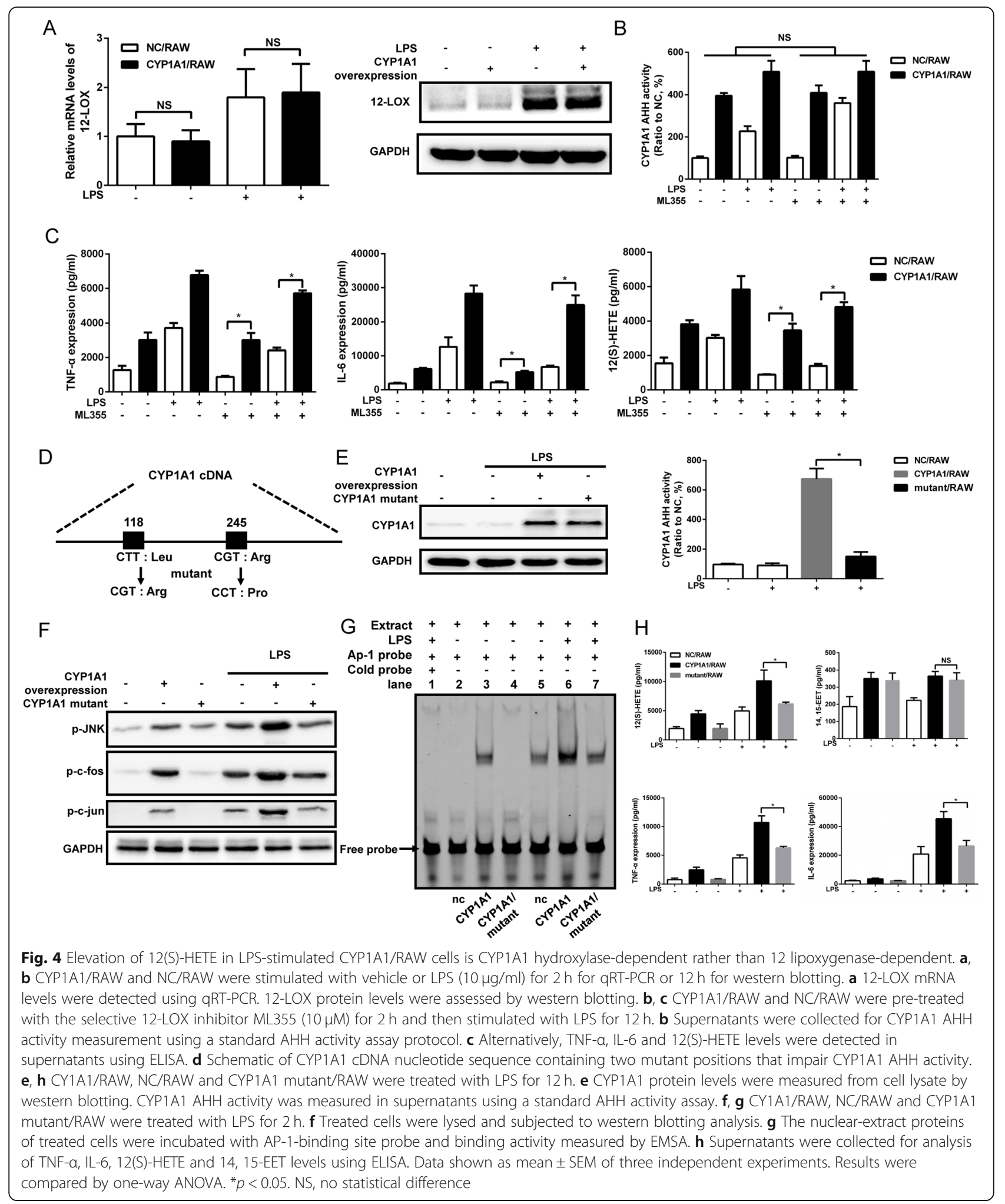

(Supplemental Figure 9 F, H). Taken together, these results indicate that CYP1A1 in macrophages plays a critical role in host defence against invading bacteria during sepsis.
The CYP1A1-12(S)-HETE-JNK-AP-1 signalling axis is activated in septic patients

Peripheral monocytes isolated from healthy individuals treated with heat-killed E. coli exhibited elevated levels 


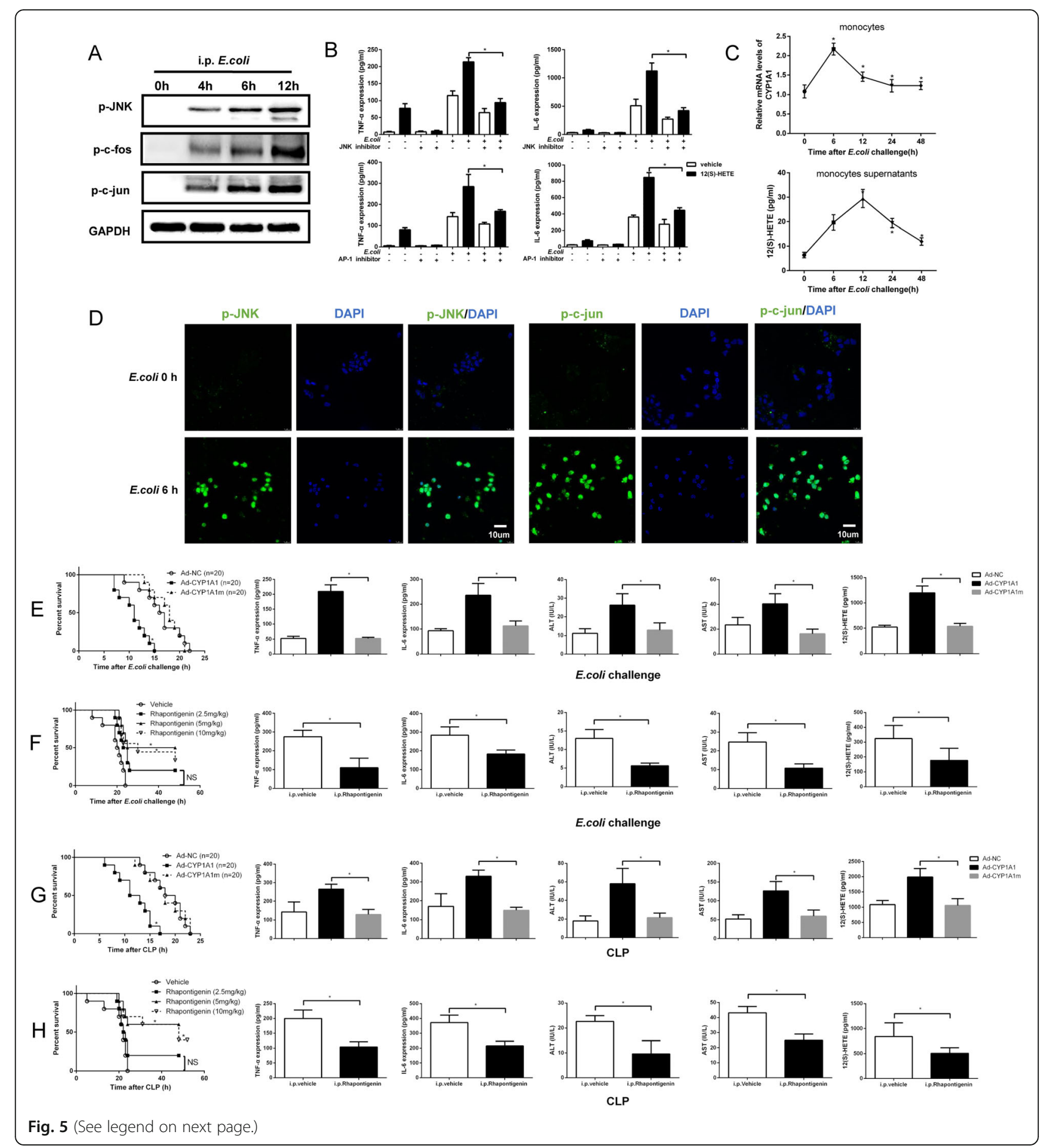


(See figure on previous page.)

Fig. 5 Regulation of the CYP1A1-12(S)HETE-JNK - AP-1 signalling axis in septic mice. a Mice were intraperitoneally injected with E. coli $\left(1.2 \times 10^{11}\right.$ CFUs $/ \mathrm{kg}$ ) at the indicated times, respectively. PMs were isolated from E. coli-treated mice and lysed for analysis of JNK/AP-1 phosphorylation. $\mathbf{b}$ Mice were pre-treated with JNK inhibitor $(30 \mathrm{mg} / \mathrm{kg})$, AP-1 inhibitor $(20 \mathrm{mg} / \mathrm{kg})$, or vehicle for $2 \mathrm{~h}$ and then stimulated with E. coli and 12(S)-HETE $(10 \mathrm{mg} / \mathrm{kg})$ for $12 \mathrm{~h}$. PLFs were extracted for analysis of TNF-a and IL-6 protein levels $(n=6)$. Results were compared by one-way ANOVA. $\mathbf{c}, \mathbf{d}$ Mice were intraperitoneally injected with E. coli. c Peripheral monocytes were isolated at the indicated times and cultured for $2 \mathrm{~h}$. Supernatants were collected for analysis of 12(S)-HETE levels using ELISA. Results were compared by one-way ANOVA. CYP1A1 mRNA levels in peripheral monocytes from E. coli-treated mice were measured by qRT-PCR. Results were compared by one-way ANOVA. $\mathbf{d}$ JNK/c-jun phosphorylation in peripheral monocytes from E. coli-treated mice was assessed using LSCM. Bar: $10 \mu \mathrm{m}$. e, g PMs transfected with Ad-NC, Ad-CYP1A1 or AdCYP1A1m were injected intraperitoneally into WT mice 2 days before E. coli or CLP impact. f, h Rhapontigenin $(2.5 \mathrm{mg} / \mathrm{kg}, 5 \mathrm{mg} / \mathrm{kg}$ and $10 \mathrm{mg} / \mathrm{kg}$ dosages were used in this experiment) were intraperitoneally injected into WT mice $1 \mathrm{~h}$ before $E$. coli or CLP impact. e-h Survival rates were monitored for 2 days after E. coli or CLP impact and presented as Kaplan-Meier survival curves. Results were compared by log-rank test $(n=20)$. PLFs and peripheral serum were collected from each group $12 \mathrm{~h}$ after $E$. coli or CLP impact and TNF-a (PLFs), IL-6 (PLFs), 12(S)-HETE (PLFs), ALT (serum) and AST (serum) levels were detected by ELISA $(n=6)$. Results were compared by one-way ANOVA. ${ }^{*} p<0.05$. NS, no statistical difference

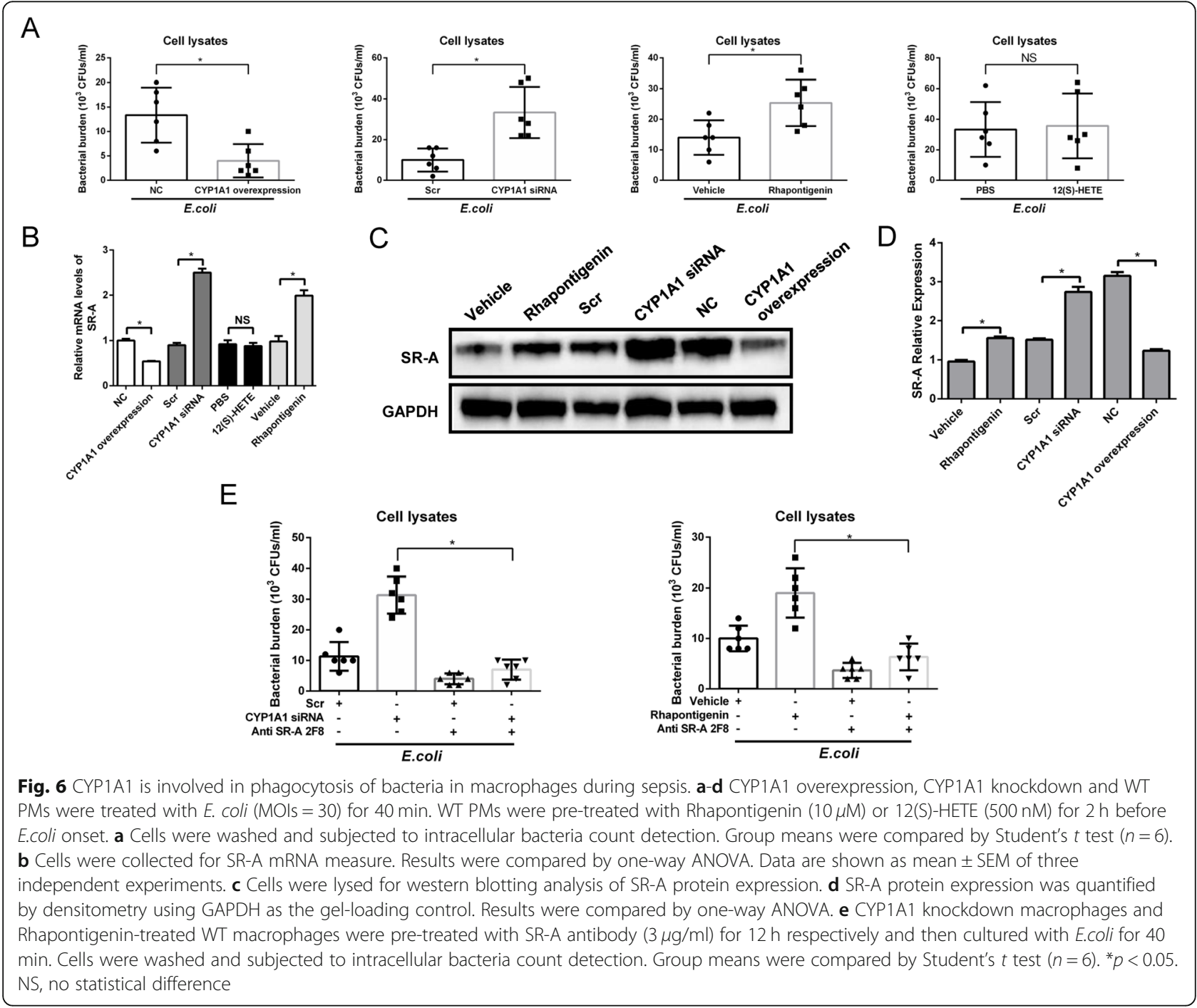


of TNF- $\alpha$, IL-6 and 12(S)-HETE (Fig. 7a). CYP1A1 protein expression levels as well as JNK and AP-1 phosphorylation levels were also increased (Fig. 7b). We then compared levels of these signalling factors between septic patients and healthy controls ( $n=30$ in each group). Consistent with preclinical results in mice, CYP1A1 mRNA levels in isolated peripheral monocytes, TNF- $\alpha$, IL-6 and 12(S)-HETE concentrations in peripheral blood plasma were significantly elevated in septic patients compared with healthy controls. More strikingly, both CYP1A1 mRNA expression levels and 12(S)-HETE concentrations in patients were significantly correlated with SOFA score (Fig. 7c). TNF- $\alpha$, IL-6 and 12(S)-HETE concentrations were also increased in peripheral monocytes (from 10 healthy and 10 septic individuals) cultured supernatants (Fig. 7d). Additionally, monocytes from 6 septic patients and 6 healthy controls were coaxed into macrophages by PMA. The elevations of CYP1A1 in monocytes-derived macrophages, 12(S)-HETE, TNF- $\alpha$ and IL- 6 in supernatants were confirmed by RT-qPCR and ELISA, respectively (Fig. 7d). Furthermore, LSCM confirmed that CYP1A1 protein expression and JNK/ AP-1 phosphorylation were enhanced in peripheral monocytes of sepsis patients (Fig. 7f). These results suggest that the CYP1A1-12(S)-HETE-JNK - AP-1 signalling axis is also hyperactivated in sepsis patients and contributes to sequential organ failure.

\section{Discussion}

In this study, we found that CYP1A1 expression was increased in PMs activated by either LPS or heat-killed $E$. coli. Surprisingly, upregulation of CYP1A1 in LPS- or $E$. coli-induced PMs was AhR-independent. The expression levels of CYP1A1 have been measured in multiple organs and cells of different inflammatory models. A previous study found that CYP1A1 mRNA levels were markedly elevated in microglia at $12 \mathrm{~h}$ after LPS treatment [37]. CYP1A1 protein levels were also increased in mouse lung and liver following incense smoke challenge [13]. Diesel exhaust particles also increased CYP1A1 mRNA expression in human bronchial epithelial cells, accompanied by pro-inflammatory factor elevation [11]. Conversely, CYP1A1 expression was suppressed in mice inflammatory Hepa cells [38, 39] and LPS-stimulated bovine mammary epithelial cells [40]. These results suggest that the relationship between CYP1A1 expression and inflammation might be species-, tissue- and cell type-specific. Here, we report for the first time that E.coli-induced CYP1A1 upregulation is AhR-independent in PMs, which is consistent with several lines of evidence. For instance, glycine methyl transferase upregulated CYP1A1 [41], while Nrf2, Oct-1 and C/EBP downregulated CYP1A1 [42, 43], but these findings had not been confirmed in inflammatory macrophages. Although AhR is considered a primary regulator of
CYP1A1, our findings suggest that CYP1A1 expression in macrophages is under the control of various transcription factors that can induces CYP1A1 overexpression in response to inflammatory stimulation.

CYP1A1 suppressed inflammatory responses in parenchymal cells, pulmonary macrophages and epithelial cells $[16,40,44,45]$, but also increased inflammatory responses in some cases. CYP1A1 was upregulated in lung and liver of incense-challenged mice, accompanied by inflammatory factor increase [13]. Further, inflammatory factor $\mathrm{LTB}_{4}$ was markedly reduced in neutrophils extracted from zymosan-challenged CYP1A1 knockout mice [20]. Systemic CYP1A1 overexpression also markedly increased mice mortality following Citrobacter rodentium infection [17], which is associated with the dysfunction of FICZAhR-IL-22 axis in $\mathrm{T}_{\mathrm{H}} 17$ cells, while we found that macrophage CYP1A1 could induce pro-inflammatory responses in an AhR-independent manner. These studies are in line with our findings, although they focused on different cells or stimuli. In the current study, TNF- $\alpha$ and IL- 6 expression levels were increased in CYP1A1-overexpressing RAW compared to NC cells following LPS stimulation, while siRNA-mediated CYP1A1 knockdown in PMs impaired TNF- $\alpha$ and IL- 6 upregulation induced by LPS.

Despite the known immune functions of AhR, there had been no systematic study investigating the role of CYP1A1 in innate immune responses. We demonstrate that pro-inflammatory stimuli induce CYP1A1 upregulation independent of $A h R$ and that this upregulation is necessary for the enhanced expression/release of proinflammatory factors TNF- $\alpha$ and IL- 6 . The function of CYP1A1 in the regulation of various inflammatory factors in other immune cell types warrants further investigation. A previous study found that JNK could be activated by an AhR-related CYP1A1 inducer PCB118 in rat thyroid FRTL-5 cells and this resulted in increased TNF- $\alpha$ and IL-6 expression [46]. Several studies have also found that AP-1 can be activated by various CYP1A1 inducers including TCDD, benzo $[a]$ pyrene $[25,47]$ betanaphthoflavone [48]. These results are in support of our findings that CYP1A1-overexpression elevated the phosphorylation levels of JNK/AP-1 and resulted in increased TNF- $\alpha$ and IL- 6 expression, while JNK knockout impeded AP-1 phosphorylation and pro-inflammatory factor release in macrophages. AP-1 activation appears to be modulated mainly by MAPK signalling pathways, but p38 phosphorylation level showed no specific association with AP-1 phosphorylation under either normal or high CYP1A1 expression following LPS stimulation. Alternatively, JNK/AP-1 knockout and MAPK expression in CYP1A1/RAW and NC/RAW cells confirmed that CYP1A1 overexpression increases TNF- $\alpha$ and IL- 6 by facilitating the phosphorylation of JNK, which then activates AP-1. Furthermore, we found that upregulated CYP1A1 


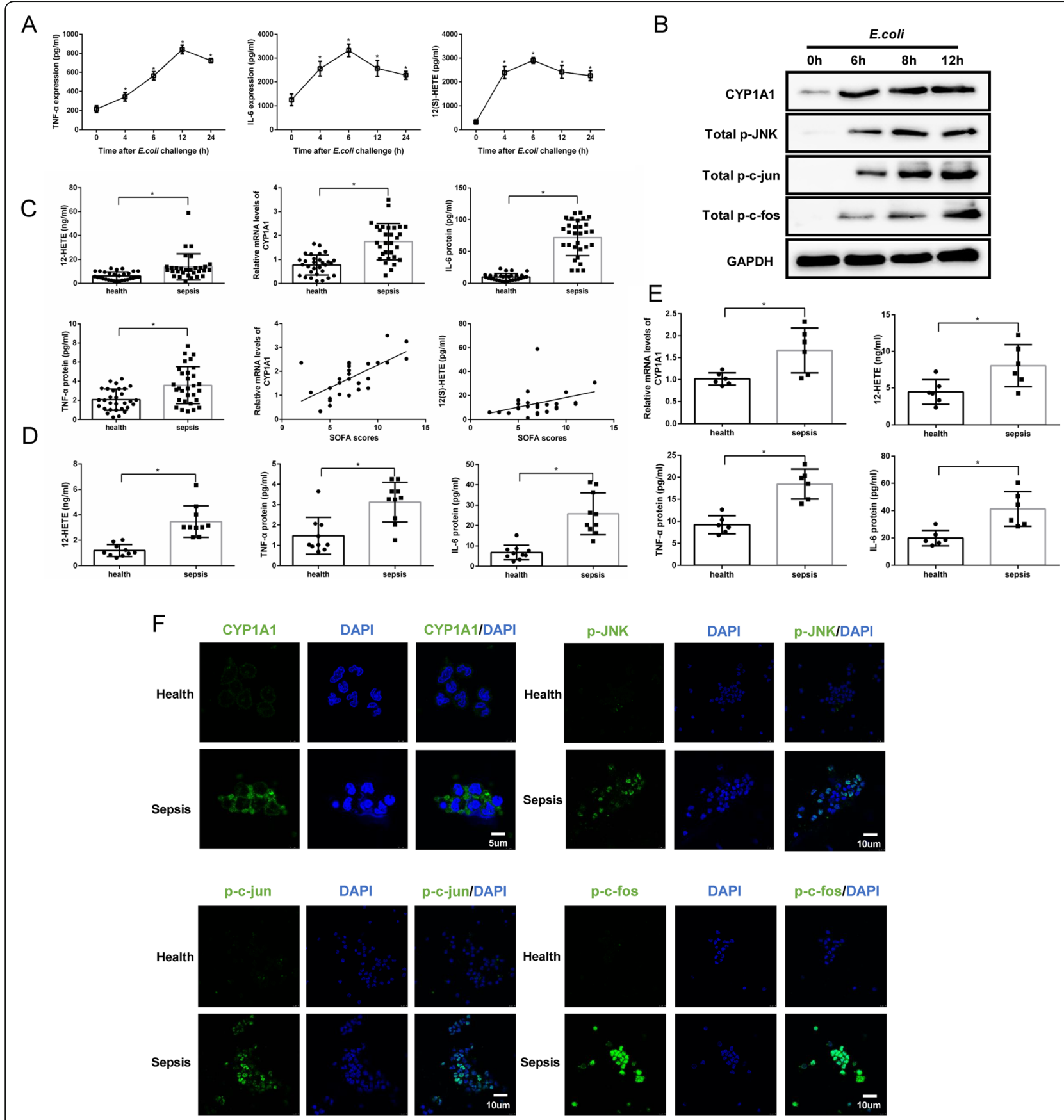

Fig. 7 The CYP1A1-12(S)-HETE-JNK-AP-1 signalling axis is activated in septic patients. $\mathbf{a}$, $\mathbf{b}$ Human peripheral monocytes were isolated from healthy donors and treated with heat-killed $E$. coli $(\mathrm{MOls}=10)$ at the indicated times. Supernatants were collected for analysis of TNF-a, IL-6 and 12(S)-HETE levels using ELISA. Cell lysates were used for JNKAP-1 phosphorylation assessment. Mean of three independent experiments. Results were compared by one-way ANOVA. c CYP1A1 mRNA levels in peripheral monocytes from the studied subjects were measured by qRT-PCR. TNFa, IL-6 and 12(S)-HETE levels in plasma were analysed by ELISA ( $n=30$ for healthy individuals, $n=30$ for sepsis patients). Group means were compared by Student's $t$ test. Correlation of SOFA scores with CYP1A1 $(r=0.65, P<0.05)$ and 12(S)-HETE $(r=0.38, P<0.05)$ in sepsis patients. $\mathbf{d}$ Peripheral monocytes were isolated from 10 septic patients and 10 healthy controls and cultured for $2 \mathrm{~h}$, respectively. Supernatants were collected for analysis of TNF-a, IL-6 and 12(S)-HETE levels using ELISA. Group means were compared by Student's $t$ test. e Peripheral monocytes were isolated from 6 septic patients and 6 healthy controls and coaxed into macrophages by PMA for $48 \mathrm{~h}$. Supernatants were collected for analysis of TNF-a, IL-6 and 12(S)-HETE levels using ELISA. CYP1A1 mRNA levels in monocytes-derived macrophages were measured by qRT-PCR. Group means were compared by Student's $t$ test. $\mathbf{f}$ CYP1A1 protein, JNK phosphorylation and AP-1 phosphorylation levels in peripheral monocytes from healthy individuals and septic patients were assessed using LSCM. Bar, CYP1A1: $5 \mu \mathrm{m}$, JNKVAP-1: $10 \mu \mathrm{m}$. Data shown as mean \pm SEM of triplicate experiments. ${ }^{*} p<0.05$ 
impacted the phagocytosis of E.coli in macrophages via decreasing the expression of SR-A for the first time, while CYP1A1 knock down or Rhapontigenin treatment enhanced the phagocytosis and SR-A expression, suggesting that the macrophage CYP1A1 also plays an important role in host defence via regulating macrophages phagocytosis and SR-A expression.

12(S)-HETE is involved in many immune-related diseases [49-54], trauma [55] and JNK/AP-1 activation [2224]. In this study, we demonstrated for the first time that levels of 12(S)-HETE were elevated in septic mice and patients, while treatment of mice with 12(S)-HETE caused more severe inflammatory responses than vehicle-treated controls. As another metabolite contributed by the epoxidase activity of CYP1A1, EETs (such as 11,12-EET) act as anti-inflammatories by suppressing NF- $\mathrm{BB}$ activation but promoting JNK/AP-1 phosphorylation [56, 57], while 12(S)-HETE acts as a pro-inflammatory factor by intensifying both NF- $\mathrm{kB}$ and JNK/AP-1 phosphorylation [22, 23, 58]. These findings may explain the reciprocal effects of CYP1A1 overexpression on NF-kB and JNK/AP-1 since EETs and 12(S)-HETE have opposite effects on NF- $k B$ but both promote JNK/AP-1 activities.
Several limitations of the current study should be addressed. Although we observed that LPS- or E. coli-induced CYP1A1 overexpression was AhR-independent, it remains unclear which transcriptional factors mediate CYP1A1 expression in $\mathrm{AhR}^{-/-}$overactivated macrophages. We observed that mRNA expression levels of Arg-1 and PPAR $\gamma$, two M2 phenotype markers, were decreased in LPS-stimulated CYP1A1/RAW cells compared with NC/RAW cells, but the details of this effect remain to be fully characterized. We also revealed that CYP1A1-associated JNK/AP-1 activation was directly regulated by 12(S)-HETE, but the mechanism of this effect remains unknown. Although it was verified that GPR31, a 12(S)-HETE-selective receptor, mediated the function of 12(S)-HETE in dermatosis [58], the specific function of GPR31 needs to be further investigated in inflammatory macrophages. In addition, it is notable that LPS acts as an inflammatory agent via binding to TLR4, while CYP1A1-induced 12(S)-HETE may act through agitating GPR31. They are two different pathways indeed but they collectively participate in the inflammatory responses via targeting JNK/AP-1 axis. The potential interaction between LPS-TLR4 axis and CYP1A1/12(S)-

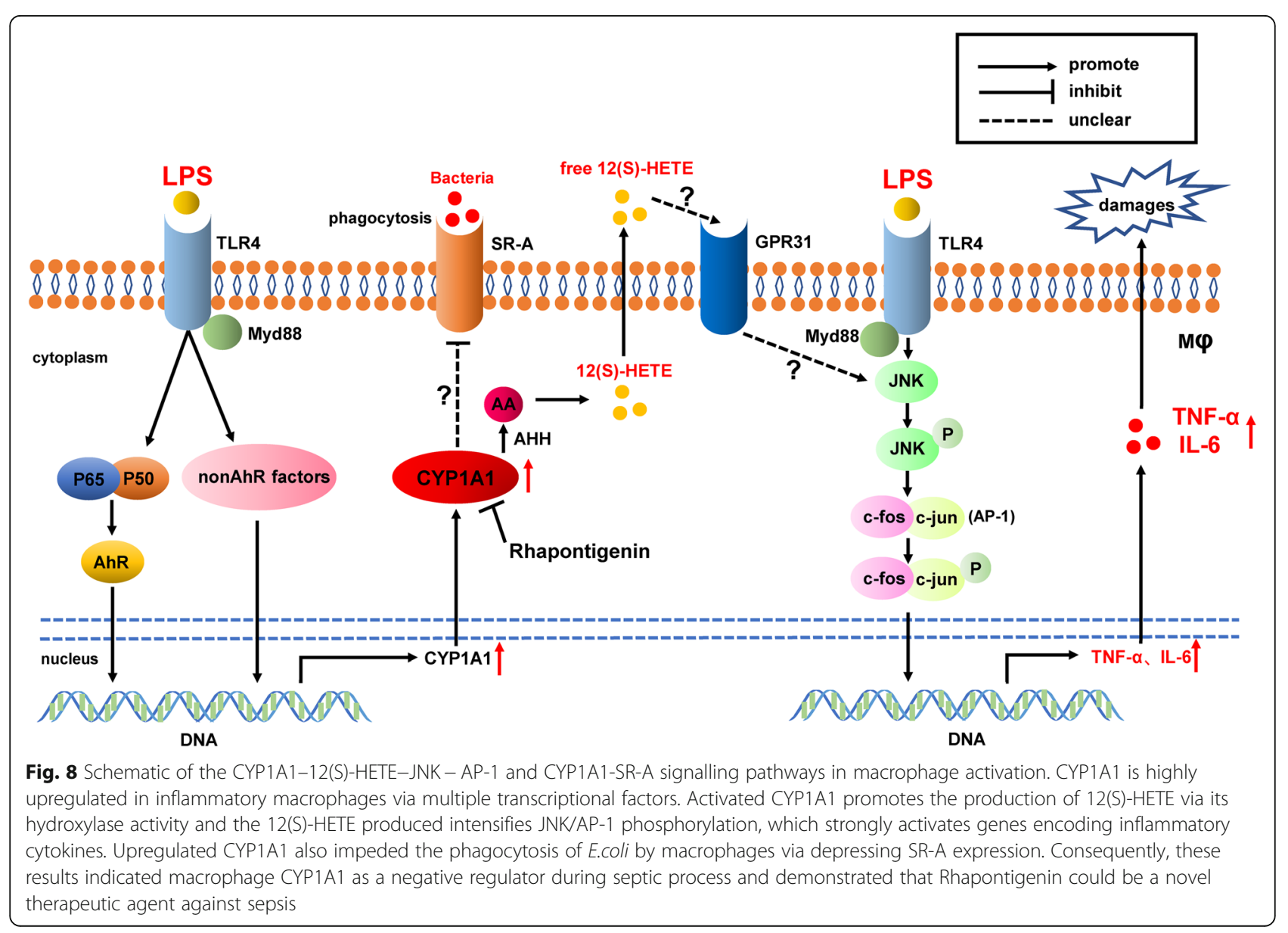


HETE-GPR31 axis needs a further assessment. Nevertheless, our study demonstrates the fundamental importance of CYP1A1 and 12(S)-HETE in overactivated macrophages.

\section{Conclusions}

In the present study, we showed that CYP1A1 was highly expressed in inflammatory macrophages and promoted TNF- $\alpha$ and IL- 6 release. The elevation of TNF- $\alpha$ and IL- 6 levels were mediated by JNK/AP-1 activation rather than by the NF-kB pathway. Excessive 12(S)HETE production mediated by overexpressed CYP1A1 was required for JNK/AP-1 activation in overactivated macrophages. Mice subjected to adoptive transfer of CYP1A1-overexpressing macrophages were more susceptible to fatal E. coli challenge, highlighting CYP1A1 as an important driver of inflammatory responses. Moreover, we found positive correlations between SOFA scores and both monocyte CYP1A1 and plasma 12(S)HETE in septic patients. Collectively, our results identify a novel therapy target, CYP1A1, regulating macrophage inflammatory responses and phagocytosis during sepsis (Fig. 8). Based on these findings, analogues of 12(S)HETE or selective antagonists of CYP1A1, especially Rhapontigenin, may be the promising treatments against inflammatory diseases and sepsis.

\section{Supplementary information}

Supplementary information accompanies this paper at https://doi.org/10. 1186/s12964-020-0523-3.

Additional file 1: Figure S1. Confirmation of transfections. Figure S2. Relative expression levels of inflammatory factors in overactivated macrophages. Figure S3. The inhibitory effects of Rhapontigenin on LPS induced TNF- $a$ and IL-6 secretion in PMs. Figure S4. Validation of the NF-KB signalling pathway and different MAPK signalling pathways in LPSstimulated CYP1A1/RAW and NC/RAW. Figure S5. The levels of 12(S)HETE in PLFs from E.coli- and CLP-induced septic mice. Figure S6. Detection of lentivirus infection rate in PMs. Figure S7. The regulation of CYP1A1-JNK-AP-1 axis in septic mice. Figure S8. Platelet count in PLFs from CYP1A1-overexpressed macrophages transferred septic mice. Figure S9. CYP1A1 is involved in phagocytosis of bacteria in macrophages during sepsis.

Additional file 2: Table S1.

Additional file 3: Table S2

\author{
Abbreviations \\ 12(S)-HETE: 12S-hydroxy-5Z, 8Z, 10E, 14Z-eicosatetraenoic acid; 12-LOX: 12- \\ lipoxygenase; AA: Arachidonic acid; AhR: Aryl hydrocarbon receptor; \\ CYP1A1: Cytochrome P450 1A1; EETs: Epoxyeicosatrienoic acids; \\ EMSA: Electrophoretic mobility shift assay; LPS: Lipopolysaccharide; \\ LSCM: Laser scanning confocal microscopy; NC: Negative control; \\ PLFs: Peritoneal lavage fluids; PMs: Peritoneal macrophages; SOFA: Sequential \\ Organ Failure Assessment; SR-A: Scavenger receptor A
}

\section{Author's contributions}

$L-x T$ and XT performed most of the experiments, analyzed the research data, and wrote the manuscript. J-yZ, LL and WZ helped with animal experiments. X-yM, W-qT, WM, XY and S-WC helped with cellular experiments. C-zL and $\mathrm{H}$ $\mathrm{pL}$ contributed to experimental design. $\mathrm{H}$-pL designed experiments, interpreted data and supervised the work. All authors read and approved the final manuscript.

\section{Funding}

This work was greatly supported by grants from the National Natural Science Foundation of China (NSFC, No. 81871612), the Fund of State Key Laboratory of Trauma, Burn, and Combined Injury (No. SKLYJF03, SKLZZ201709) and the Fund of Basic Science and Frontier Q8 Technology Research of Chongqing (cstc2017jcyjAX0159).

\section{Availability of data and materials}

The datasets used and/or analysed during the current study are available from the corresponding author on reasonable request.

\section{Ethics approval and consent to participate}

This study was approved by the Institute Research Medical Ethics Committee of Daping Hospital of Army Medical University. All the individuals were participating in this study with written informed consent. All animalstreatment operations were executed according to the Army Medical University Ethical Guidelines for Animal Experiment.

\section{Consent for publication}

The study was undertaken with Army Medical University.

\section{Competing interests}

The authors declare that they have no competing interests.

\section{Author details}

${ }^{1}$ State Key Laboratory of Trauma, Burns and Combined Injury, Department of Wound Infection and Drug, Daping Hospital, Army Medical University, Yuzhong District, Chongqing, China. ${ }^{2}$ Trauma Center, The First Affiliated Hospital of Hainan Medical University, Haikou, China. ${ }^{3}$ Emergency and Trauma College of Hainan Medical University, Haikou, China.

Received: 12 November 2019 Accepted: 29 January 2020

Published online: 04 May 2020

References

1. Vincent JL, Opal SM, Marshall JC, et al. Sepsis definitions: time for change. Lancet. 2013;381(9868):774-5.

2. Lelubre $C$, Vincent JL. Mechanisms and treatment of organ failure in sepsis. Nat Rev Nephrol. 2018;14(7):417-27.

3. Murray PJ, Allen JE, Biswas SK, et al. Macrophage activation and polarization: nomenclature and experimental guidelines. Immunity. 2014;41(1):14-20.

4. Rosadini CV, Kagan JC. Early innate immune responses to bacterial LPS. Curr Opin Immunol. 2017:44:14-9.

5. Hoshino K, Takeuchi O, Kawai T, et al. Cutting edge: toll-like receptor 4 (TLR4)-deficient mice are hyporesponsive to lipopolysaccharide: evidence for TLR4 as the Lps gene product. J Immunol. 1999;162(7):3749-52.

6. Takeuchi O, Hoshino K, Kawai T, et al. Differential roles of TLR2 and TLR4 in recognition of gram-negative and gram-positive bacterial cell wall components. Immunity. 1999;11(4):443-51.

7. Underhill DM, Goodridge HS. Information processing during phagocytosis. Nat Rev Immunol. 2012;12(7):492-502.

8. Danielson PB. The cytochrome P450 superfamily: biochemistry, evolution and drug metabolism in humans. Curr Drug Metab. 2002;3(6):561-97.

9. Watkins PB. Role of cytochromes P450 in drug metabolism and hepatotoxicity. Semin Liver Dis. 1990;10(4):235-50.

10. Zuber R, Anzenbacherova E, Anzenbacher P. Cytochromes P450 and experimental models of drug metabolism. J Cell Mol Med. 2002;6(2):189-98.

11. Totlandsdal Al, Cassee FR, Schwarze P, et al. Diesel exhaust particles induce CYP1A1 and pro-inflammatory responses via differential pathways in human bronchial epithelial cells. Part Fibre Toxicol. 2010;7:41.

12. Zhang $Y$, Liu $X$, McHale $C$, et al. Bone marrow injury induced via oxidative stress in mice by inhalation exposure to formaldehyde. PLoS One. 2013;8(9):e74974. 
13. Hussain T, Al-Attas OS, Al-Daghri NM, et al. Induction of CYP1A1, CYP1A2, CYP1B1, increased oxidative stress and inflammation in the lung and liver tissues of rats exposed to incense smoke. Mol Cell Biochem. 2014;391(1-2):127-36.

14. Yang T, Jia Y, Ma Y, et al. Comparative proteomic analysis of Bleomycininduced pulmonary fibrosis based on isobaric tag for quantitation. Am J Med Sci. 2017;353(1):49-58.

15. Lingappan $K$, Jiang $W$, Wang $L$, et al. Sex-specific differences in hyperoxic lung injury in mice: implications for acute and chronic lung disease in humans. Toxicol Appl Pharmacol. 2013;272(2):281-90.

16. Fang $\mathrm{X}$, Zhao W, Xu J, et al. CYP1A1 mediates the suppression of major inflammatory cytokines in pulmonary alveolar macrophage (PAM) cell lines caused by mycoplasma hyponeumoniae. Dev Comp Immunol. 2016;65:132-8.

17. Schiering C, Wincent E, Metidji A, et al. Feedback control of AHR signalling regulates intestinal immunity. Nature. 2017;542(7640):242-5.

18. Nguyen $\mathrm{CH}$, Brenner $\mathrm{S}$, Huttary $\mathrm{N}$, et al. AHR/CYP1A1 interplay triggers lymphatic barrier breaching in breast cancer spheroids by inducing 12(S)HETE synthesis. Hum Mol Genet. 2016;25(22):5006-16.

19. El-Sherbeni AA, El-Kadi AO. Repurposing resveratrol and fluconazole to modulate human cytochrome P450-mediated Arachidonic acid metabolism. Mol Pharm. 2016;13(4):1278-88.

20. Divanovic S, Dalli J, Jorge-Nebert LF, et al. Contributions of the three CYP1 monooxygenases to pro-inflammatory and inflammation-resolution lipid mediator pathways. J Immunol. 2013;191(6):3347-57.

21. Guha M, Mackman N. LPS induction of gene expression in human monocytes. Cell Signal. 2001;13(2):85-94.

22. Lysz TW, Arora JK, Lin C, et al. 12(S)-hydroxyeicosatetraenoic acid regulates DNA synthesis and protooncogene expression induced by epidermal growth factor and insulin in rat lens epithelium. Cell Growth Differ. 1994;5(10):1069-76.

23. Bleich $D, C$ hen $S$, Wen $Y$, et al. The stress-activated c-Jun protein kinase (JNK) is stimulated by lipoxygenase pathway product 12-HETE in RIN m5F cells. Biochem Biophys Res Commun. 1997;230(2):448-51.

24. Zhang XJ, Cheng X, Yan ZZ, et al. An ALOX12-12-HETE-GPR31 signaling axis is a key mediator of hepatic ischemia-reperfusion injury. Nat Med. 2018; 24(1):73-83.

25. Puga A, Barnes SJ, Chang $C$, et al. Activation of transcription factors activator protein-1 and nuclear factor-kappaB by 2,3,7,8-tetrachlorodibenzo-p-dioxin. Biochem Pharmacol. 2000;59(8):997-1005.

26. Kimura S, Smith HH, Hankinson O, et al. Analysis of two benzo [a]pyreneresistant mutants of the mouse hepatoma Hepa-1 P(1)450 gene via cDNA expression in yeast. EMBO J. 1987;6(7):1929-33.

27. Nemoto N, Sakurai J. Activation of Cypla1 and Cyp1a2 genes in adult mouse hepatocytes in primary culture. Jpn J Cancer Res. 1993;84(3):272-8.

28. Hou J, Chen Q, Wu X, et al. S1PR3 signaling drives bacterial killing and is required for survival in bacterial Sepsis. Am J Respir Crit Care Med. 2017; 196(12):1559-70

29. Taylor FJ, Wada H, Kinasewitz G. Description of compensated and uncompensated disseminated intravascular coagulation (DIC) responses (non-overt and overt DIC) in baboon models of intravenous and intraperitoneal Escherichia coli sepsis and in the human model of endotoxemia: toward a better definition of DIC. Crit Care Med. 2000;28(9 Suppl):S12-9.

30. Ma Q, Lu AY. CYP1A induction and human risk assessment: an evolving tale of in vitro and in vivo studies. Drug Metab Dispos. 2007;35(7):1009-16.

31. Chun YJ, Ryu SY, Jeong TC, et al. Mechanism-based inhibition of human cytochrome P450 1A1 by rhapontigenin. Drug Metab Dispos. 2001:29(4 Pt 1):389-93.

32. Shimada T, Yamazaki $H$, Foroozesh $M$, et al. Selectivity of polycyclic inhibitors for human cytochrome P450s 1A1, 1A2, and 1B1. Chem Res Toxicol. 1998;11(9):1048-56.

33. Hamberg M, Samuelsson B. Prostaglandin endoperoxides. Novel transformations of arachidonic acid in human platelets. Proc Natl Acad Sci U S A. 1974:71(9):3400-4

34. Nugteren $\mathrm{DH}$. Arachidonate lipoxygenase in blood platelets. Biochim Biophys Acta. 1975;380(2):299-307.

35. Luci DK, Jameson JN, Yasgar A, et al. Synthesis and structure-activity relationship studies of 4-((2-hydroxy-3-methoxybenzyl)amino) benzenesulfonamide derivatives as potent and selective inhibitors of 12 lipoxygenase. J Med Chem. 2014;57(2):495-506.

36. Peiser L, Gough PJ, Kodama T, et al. Macrophage class a scavenger receptor-mediated phagocytosis of Escherichia coli: role of cell heterogeneity, microbial strain, and culture conditions in vitro. Infect Immun. 2000;68(4):1953-63.
37. Lee $\mathrm{YH}$, Lin $\mathrm{CH}$, Hsu PC, et al. Aryl hydrocarbon receptor mediates both proinflammatory and anti-inflammatory effects in lipopolysaccharideactivated microglia. GLIA. 2015;63(7):1138-54.

38. Morgan ET. Regulation of cytochrome $\mathrm{p} 450$ by inflammatory mediators: why and how? Drug Metab Dispos. 2001;29(3):207-12.

39. Santes-Palacios R, Ornelas-Ayala D, Cabanas N, et al. Regulation of human cytochrome P4501A1 (hCYP1A1): a plausible target for chemoprevention? Biomed Res Int. 2016;2016:5341081.

40. Zhang WY, Wang H, Qi S, et al. CYP1A1 relieves lipopolysaccharide-induced inflammatory responses in bovine mammary epithelial cells. Mediat Inflamm. 2018:2018:4093285.

41. Bhat R, Bresnick E. Glycine N-methyltransferase is an example of functional diversity. Role as a polycyclic aromatic hydrocarbon-binding receptor. J Biol Chem. 1997;272(34):21221-6.

42. Wu Z, Uchi H, Morino-Koga S, et al. Z-Ligustilide inhibits benzo(a)pyreneinduced CYP1A1 upregulation in cultured human keratinocytes via ROSdependent Nrf2 activation. Exp Dermatol. 2014;23(4):260-5.

43. Sidorova YA, Perepechaeva ML, Pivovarova EN, et al. Menadione suppresses Benzo (alpha)pyrene-induced activation of cytochromes P450 1A: insights into a possible molecular mechanism. PLoS One. 2016;11(5):e155135.

44. Salnikova LE, Smelaya TV, Golubev AM, et al. CYP1A1, GCLC, AGT, AGTR1 gene-gene interactions in community-acquired pneumonia pulmonary complications. Mol Biol Rep. 2013;40(11):6163-76.

45. Lingappan $K$, Jiang W, Wang $L$, et al. Mice deficient in the gene for cytochrome P450 (CYP)1A1 are more susceptible than wild-type to hyperoxic lung injury: evidence for protective role of CYP1A1 against oxidative stress. Toxicol Sci. 2014;141(1):68-77.

46. Xu B, Yang $H$, Sun $M$, et al. 2,3',4,4',5-Pentachlorobiphenyl induces inflammatory responses in the thyroid through JNK and aryl hydrocarbon receptor-mediated pathway. Toxicol Sci. 2016;149(2):300-11.

47. Hoffer A, Chang CY, Puga A. Dioxin induces transcription of fos and Jun genes by ah receptor-dependent and -independent pathways. Toxicol Appl Pharmacol. 1996:141(1):238-47.

48. Carriere V, Rodolosse A, Lacasa M, et al. Hypoxia and CYP1A1 inductiondependent regulation of proteins involved in glucose utilization in Caco-2 cells. Am J Phys. 1998;274(6):G1101-8.

49. Munoz-Garcia A, Thomas CP, Keeney DS, et al. The importance of the lipoxygenase-hepoxilin pathway in the mammalian epidermal barrier. Biochim Biophys Acta. 2014;1841(3):401-8.

50. Voorhees JJ. Leukotrienes and other lipoxygenase products in the pathogenesis and therapy of psoriasis and other dermatoses. Arch Dermatol. 1983;119(7):541-7.

51. Wollard PM, Cunnigham FM, Murphy GM, et al. A comparison of the proinflammatory effects of 12(R)- and 12(S)-hydroxy-5,8,10,14eicosatetraenoic acid in human skin. Prostaglandins. 1989;38(4):465-71.

52. Kragballe K, Voorhees JJ. Arachidonic acid in psoriasis. Pathogenic role and pharmacological regulation. Acta Derm Venereol Suppl (Stockh). 1985;120: 12-7.

53. Fretland DJ, Anglin CP, Bremer $M$, et al. Antiinflammatory effects of secondgeneration leukotriene B4 receptor antagonist, SC-53228: impact upon leukotriene B4- and 12(R)-HETE-mediated events. INFLAMMATION. 1995; 19(2):193-205.

54. Fox T, Gotlinger KH, Dunn MW, et al. Dysregulated heme oxygenase-ferritin system in pterygium pathogenesis. Cornea. 2013;32(9):1276-82

55. Farias SE, Heidenreich KA, Wohlauer MV, et al. Lipid mediators in cerebral spinal fluid of traumatic brain injured patients. J Trauma. 2011;71(5):1211-8.

56. Deng Y, Theken KN, Lee CR. Cytochrome P450 epoxygenases, soluble epoxide hydrolase, and the regulation of cardiovascular inflammation. J Mo Cell Cardiol. 2010:48(2):331-41.

57. Li P, Lahvic JL, Binder $V$, et al. Epoxyeicosatrienoic acids enhance embryonic haematopoiesis and adult marrow engraftment. Nature. 2015;523(7561): 468-71.

58. Guo Y, Zhang W, Giroux C, et al. Identification of the orphan G proteincoupled receptor GPR31 as a receptor for 12-(S)-hydroxyeicosatetraenoic acid. J Biol Chem. 2011;286(39):33832-40.

\section{Publisher's Note}

Springer Nature remains neutral with regard to jurisdictional claims in published maps and institutional affiliations. 